\title{
LA AUDITORÍA COMO FUNDAMENTO EN EL CONTROL DE INVENTARIOS EN LAS MICRO Y PEQUEÑAS EMPRESAS
}

\author{
AUDIT AS A BASIS IN THE INVENTORY CONTROL IN MICRO AND SMALL \\ BUSINESSES
}

\author{
${ }^{1}$ Pedro Ricardo Quiroz Quezada
}

\begin{abstract}
Resumen
La presente investigación tiene como objetivo identificar y analizar todos los distintos procedimientos contables para implementar un eficiente control de inventarios con el fin de sistematizarlos a fin de resolverlos con prontitud, economizando horas-hombre tanto en los empleados como en la Alta Dirección de las empresas. Para lograr este objetivo la investigación se hizo mediante una encuesta a 60 contadores, gerentes y administradores de las Mype en Lima Metropolitana.

El análisis de los resultados llega a la siguientesconclusiones finales: A nivel de micro y pequeñas empresas, se hace pertinente la sistematización de los procesos contables con la finalidad de optimizar la administración y control de inventarios, asimismo, la eficacia, eficiencia y prontitud de los requerimientos solicitados por las distintas unidades operativas y gerentes, permitiendo la atención de actividades de mayor trascendencia empresarial.
\end{abstract}

Palabras claves: Procedimientos, inventarios, sistematización, eficacia y eficiencia.

\begin{abstract}
This researchaims to identifyand analyze all thedifferentaccountingprocedures to implementanefficien.

0tinventorycontrolinordertosystematizeresolvepromptly, saving man hoursbothemployeesandtopmanagementofcompa nies. To achieve this goal the research was done through a survey of 60 accountants, managers and administators of small business in Metropolitan Lima.

Theanalysis of the resultsreached the followingconclusions: At the level of micro and small business, thesystematization of theAccountingProcessin order tooptimize the managementand inventory controlbecomesrelevant,alsothe effectiveness, efficiency and timeliness ofthe requirements requested bythe various operating units and executives, allowing the activities of senior care business significance.
\end{abstract}

Key words: Procedures, inventories,systematization, effectiveness and efficiency.

\section{Introducción}

Actualmente en las empresas del país, existen una serie de actividades administrativas y de proyectos, que diariamente tienen que resolverse y por lo mismo generan una serie de procesos contables.

Sin embargo, observamos que muchas veces por falta de tiempo, paradigmas, $\mathrm{u}$ otras prioridades, hemos dejado de analizar la naturaleza de estos procesos, así como de sistematizar su recurrencia y cotidianeidad.

Igualmente, tampoco hemos analizado que la solución de estos problemas o procedimientos, tienen derroteros estructurables y sistematizables, que en buena cuenta, agilizarían su proceso de contabilización y archivamiento, así como descongestionarían los ámbitos administrativos de estas unidades productivas.

Esta situación hace que los empleados le dediquen gran parte de su tiempo, al seguimiento de las actividades administrativas y sus documentos de respaldo, burocratizando el área contable, y resultando engorroso y lento como instrumento de control, seguimiento y toma de decisiones, o simplemente no se hagan los procesos contables completos, en vista de considerarse micro y pequeñas empresas, lo que coadyuva a que no se tenga la información necesaria para la toma de decisiones adecuada para su crecimiento.

Es así entonces, como los empresarios de Lima y los empleados administrativos, invierten buena parte de su tiempo al seguimiento de los problemas administrativos y contables que esta situación genera, dejando de lado o postergando actividades de mayor trascendencia organizacional. Como estar en contacto con el mundo exterior, para la utilización de una mejor tecnología y capacitación del personal.

El objetivo general de la investigación es ejecutar la auditoria a través de la sistematización de los procesos contables de manera que permita optimizar 
el control interno de la documentación contable para la administración y control de los inventarios. Este objetivo general se desglosa en los siguientes objetivos específicos: a) Determinar los problemas fuentes, de cada uno de los requerimientos del control de inventarios, b) Determinar las posibilidades de resolución de cada uno de los problemas prototipos o recurrentes, c) Ejecutar todos los requerimientos contables de control de inventarios que soliciten los empresarios, d) Generar un modelo de atención sistematizado de cada uno de los procesos contables en la administración y control de inventarios.

\section{Materiales y métodos}

\section{Tipo de investigación.}

La presente investigación ha seguido las pautas de tipo descriptivo, ya que permite indagar y especificar aspectos y situaciones importantes que inciden en la necesidad de desarrollar la presente investigación.

\section{Diseño de la investigación}

El nivel de la investigación es correlacional, permite analizar el objetivo en la relación entre la sistematización de los procesos contables, optimización del control interno y la eficiencia, eficacia y prontitud de los requerimientos para la administración y control de inventarios.

\section{Población y muestra.}

Se realizó una encuesta y se entrevistóa una muestra de 60 contadores, administradores y gerentes de las pequeñas y micro empresas de lima metropolitana. La muestra fue calculada con $95.5 \%$ de confianza y un margen de error del $10 \%$ de una población de 4,480 contadores públicos, gerentes y administradores de las Micro y pequeñas empresas.

\section{Limitaciones del estudio}

Este trabajo de investigación presenta varias limitaciones: La primera limitación es el lugar únicamente en el departamento de lima y dentro de este en Lima Metropolitana.

La segunda limitación fue el tipo de participantes. El estudio se limitó a una encuesta de solo 60 contadores, gerentes y administradores, que gentilmente estuvieron dispuestos a colaborar con la realización de la investigación. Asimismo participaron como encuestadores y entrevistadores 4 estudiantes de la carrera de Ingeniería en Gestión Empresaria.

Ha sido una limitación importante la poca información que tienen las Mype en este tipo de investigación.

\section{Resultados y discusión \\ ANTECEDENTES TEÓRICOS DE LA INVESTIGACIÓN}

Revisando la bibliografía disponible, se ha encontrado vastas obras de control interno, así como algunas investigaciones, que de alguna manera podrían servir de base para este trabajo, y de los cuales daremos cuenta más adelante. Sin embargo hasta la fecha, no se ha realizado este tipo de trabajo en nuestro país, desde la óptica y perspectiva de la empresa, por lo que lo consideramos un tema inédito.

Para Senge (1998), una organización aprendiente se caracteriza por el estimulo constante de sus miembros para que aumenten sus capacidades, es decir, para que aprendan. Una organización capaz de convertir rápidamente las nuevas tecnologías en nuevos productos, servicios, procesos o procedimientos.

Según Drucker (2007), los directivos deberán aprender a poner en cuestión sus productos, servicios, procesos y procedimientos de sus propias organizaciones, y deberían estimular la creatividad de sus empleados, fomentando la mejora continua, con el fin de aprender a generar nuevos conocimientos así como generar nuevas aplicaciones de sus conocimientos presentes.

En la organización aprendiente la idea de que los directivos piensan y los empleados actúan, debe ser sustituida por una en la que se produzca la integración de pensamiento y actuación en todos los niveles de la organización, El énfasis no debe ponerse en el proceso o almacenamiento de la información, sino en la generación, aplicación y distribución de conocimiento.

Barragán (2012),afirma que la administración efectiva de las nuevas Mypes latinoamericanas requiere de un conocimiento profundo de varias áreas en las que estas empresas enfrentan problemas y retos como resultado de la globalización. Entre otras las áreas más importantes se encuentran en la gerencia y la planeacion estratégica, mercadotecnia, recursos humanos, análisis financiero y evaluación de proyectos, comportamiento organizacional, el comercio virtual y la adopción de tecnología.

Por lo tanto, está implícito que en el límite del sistema habrá algún proceso de toma de decisiones que tiene autoridad para utilizar los recursos disponibles para ese sistema con el fin de lograr el propósito dentro de las expectativas de desempeño especificadas y dentro de las restricciones externas al control de ese proceso de toma de decisiones. Como este modelo es general por completo, será aplicable a cada nivel de resolución en la jerarquía de sistemas. Habiendo especificado el nivel en que se define "el sistema", el conjunto de actividades dentro del límite se vuelven sus "subsistemas". En este nivel, cada subsistema puede extenderse justo de la misma manera, mediante el mecanismo de una definición raíz. Por tanto, cada modelo de sistemas en cada nivel de resolución contiene actividades de control relevantes para el proceso de transformación particular. 
La contabilidad es un sistema adaptado para clasificar los hechos económicos que ocurren en un negocio. De tal manera que, se convierte en el eje central para llevar a cabo diversos procedimientos que conducirán a la obtención del máximo rendimiento económico que implica el constituir una empresa determinada.

\section{Sistematización de los Procesos Contables}

La contabilidad, es una herramienta empresarial que permite el registro y control sistemático de todas las operaciones que se realizan en la empresa, por ende no existe una definición concreta de la contabilidad aunque todas estas definiciones tienen algo en común.

A continuación se presentan varias acepciones de la contabilidad que han sido definidas por diferentes autores y cuerpos colegiados de la profesión contable:

"La contabilidad es el arte de registrar, clasificar y resumir en forma significativa y en términos de dinero, las operaciones y los hechos que son cuando menos de carácter financiero, así como el de interpretar sus resultados". (Instituto Americano de Contadores Públicos Certificados).

Holmes, A. (1994), señala que:"La contabilidad es el sistema que mide las actividades del negocio, procesa esa información convirtiéndola en informes y comunica estos hallazgos a los encargados de tomar las decisiones".

Meigs, R. (1994) nos dice que: "La contabilidad es el arte de interpretar, medir y describir la actividad económica".

Hidalgo, J. (2010), nos dice que: "La contabilidad es el lenguaje que utilizan los empresarios para poder medir y presentar los resultados obtenidos en el ejercicio económico, la situación financiera de las empresas, los cambios en la posición financiera y/o en el flujo de efectivo".

Redondo, A. (2012) nos dice que: "La contabilidad tiene diversas funciones, pero su principal objetivo es suministrar, cuando sea requerida o en fechas determinadas, información razonada, en base a registros técnicos, de las operaciones realizadas por un ente público o privado".

La contabilidad es una técnica que se ocupa de registrar, clasificar y resumir las operaciones mercantiles de un negocio con el fin de interpretar sus resultados, para que los gerentes a través de ella puedan orientarse sobre el curso que siguen sus negocios mediante datos contables; permitiendo así conocer la estabilidad, la solvencia de la compañía y la capacidad financiera de la empresa.

\section{Concepto de Sistema de Información Contable}

Un sistema de información contable comprende los métodos, procedimientos y recursos utilizados por una entidad para llevar un control de las actividades financieras y resumirlas en forma útil para la toma de decisiones.
La información contable se puede clasificar en dos grandes categorías: la contabilidad financiera o la contabilidad externa y la contabilidad de costos o contabilidad interna. La contabilidad financiera muestra la información que se facilita al público en general, y que no participa en la administración de la empresa, como son los accionistas, los acreedores, los clientes, los proveedores, los analistas financieros, entre otros, aunque esta información también es de mucho interés para los administradores y directivos de la empresa. Esta contabilidad permite obtener información sobre la posición financiera de la empresa, su grado de liquidez y sobre la rentabilidad de la empresa.

\section{Propósito y Naturaleza de la Información Contable}

El propósito de la contabilidad es proporcionar información financiera sobre una entidad económica. Quienes toman las decisiones administrativas necesitan de esa información financiera de la empresa para realizar una buena planeación y control de las actividades de la organización.

El papel del sistema contable de la organización es desarrollar y comunicar esta información. Para lograr estos objetivos se puede hacer uso de computadores, como también de registros manuales e informes impresos.

\section{Estructura de un Sistema Contable}

Un sistema de información contable sigue un modelo básico y un sistema de información bien diseñado, ofreciendo así control, compatibilidad, flexibilidad y una relación aceptable de costo / beneficio.

El sistema contable de cualquier empresa independientemente del sistema contable que utilicé, se deben ejecutar tres pasos básicos relacionados con las actividades financieras; los datos se deben registrar, clasificar y resumir, sin embargo el proceso contable involucra la comunicación a quienes estén interesados y la interpretación de la información contable para ayudar en la toma de decisiones comerciales.

Registro de la actividad financiera: en un sistema contable se debe llevar un registro sistemático de la actividad comercial diaria en términos económicos. En una empresa se llevan a cabo todo tipo de transacciones que se pueden expresar en términos monetarios y que se deben registrar en los libros de contabilidad. Una transacción se refiere a una acción terminada más que a una posible acción a futuro. Ciertamente, no todos los eventos comerciales se pueden medir y describir objetivamente en términos monetarios.

Clasificación de la información: un registro completo de todas las actividades comerciales implica comúnmente un gran volumen de datos, demasiado grande y diverso para que pueda ser útil para las personas encargadas de tomar decisiones. Por tanto, la información de debe 
clasificar en grupos o categorías. Se deben agrupar aquellas transacciones a través de las cuales se recibe o paga dinero.

Resumen de la información: para que la información contable utilizada por quienes toman decisiones, esté debidamente resumida. Por ejemplo, una relación completa de las transacciones de venta de una empresa como SAGA sería demasiado larga para que cualquier persona se dedicara a leerla. Los empleados responsables de comprar mercancías necesitan la información de las ventas resumidas por producto. Los gerentes de almacén necesitaran la información de ventas resumida por departamento, mientras que la alta gerencia de SAGA necesitará la información de ventas resumida por almacén.

Estos tres pasos que se han descrito: registro, clasificación y resumen constituyen los medios que se utilizan para crear la información contable. Sin embargo, el proceso contable incluye algo más que la creación de información, también involucra la comunicación de esta información a quienes estén interesados y la interpretación de la información contable para ayudar en la toma de decisiones comerciales. Un sistema contable debe proporcionar información a los gerentes y también a varios usuarios externos que tienen interés en las actividades financieras de la empresa.

\section{Utilización de la Información Contable}

La contabilidad va más allá del proceso de creación de registros e informes. El objetivo final de la contabilidad es la utilización de esta información, su análisis e interpretación. Los contadores se preocupan de comprender el significado de las cantidades que obtienen. Buscan la relación que existe entre los eventos comerciales y los resultados financieros; estudian el efecto de diferentes alternativas, por ejemplo la compra o el arriendo de un nuevo edificio; y buscan las tendencias significativas que sugieren lo que puede ocurrir en el futuro.

Si los gerentes, inversionistas, acreedores o empleados gubernamentales van a darle un uso eficaz a la información contable, también deben tener un conocimiento acerca de cómo obtuvieron esas cifras y lo que ellas significan. Una parte importante de esta comprensión es el reconocimiento claro de las limitaciones de los informes de contabilidad. Un gerente comercial u otra persona que esté en posición de tomar decisiones y que carezca de conocimientos de contabilidad, probablemente no apreciara hasta qué punto la información contable se basa en estimativos más que en mediciones precisas y exactas.

\section{Características de un sistema de información contable efectivo}

Un sistema de información bien diseñado ofrece control, compatibilidad, flexibilidad y una relación aceptable de costo / beneficio.

Control: un buen sistema de contabilidad le da a la administración control sobre las operaciones de la empresa. Los controles internos son los métodos y procedimientos que usa un negocio para autorizar las operaciones, proteger sus activos y asegurar la exactitud de sus registros contables.

Compatibilidad: un sistema de información cumple con la pauta de compatibilidad cuando opera sin problemas con la estructura, el personal, y las características especiales de un negocio en particular.

\section{Objetivos de la Información Contable}

La información contable debe servir fundamentalmente para:

Conocer y demostrar los recursos controlados por un ente económico, las obligaciones que tenga de transferir recursos a otros entes, los cambios que hubieren experimentado tales recursos y el resultado obtenido en el periodo.

Predecir flujos de efectivo.

Apoyar a los administradores en la planeación, organización y dirección de los negocios.

Tomar decisiones en materia de inversiones y crédito.

Evaluar la gestión de los administradores del ente económico.

Ejercer control sobre las operaciones del ente económico.

Fundamentar la determinación de cargas tributarias, precios y tarifas.

Ayudar a la conformación de la información estadística nacional.

Contribuir a la evaluación del beneficio o impacto social que la actividad económica representa para la comunidad.

\section{Cualidades de la Información Contable}

Para poder satisfacer adecuadamente sus objetivos, la información contable debe ser comprensible, útil y en ciertos casos se requiere que además la información sea comparable.

La información es comprensible cuando es clara y fácil de comprender.

La información es útil cuando es pertinente y confiable.

La información es pertinente cuando posee el valor de realimentación, valor de predicción y es oportuna.

La información es confiable cuando es neutral, verificable y en la medida en la cual represente fielmente los hechos económicos.

Importancia de la contabilidad en función de los usuarios de la información

La contabilidad es de gran importancia porque todas las empresas tienen la necesidad de llevar un control 
de sus negociaciones mercantiles y financieras. Así obtendrá mayor productividad y aprovechamiento de su patrimonio. Por otra parte, los servicios aportados por la contabilidad son imprescindibles para obtener información de carácter legal.

La gente que participa en el mundo de los negocios: propietarios, gerentes, banqueros, corredores de bolsa, inversionistas utilizan los términos y los conceptos contables para describir los recursos y las actividades de todo negocio, sea grande o pequeño. Aunque la contabilidad ha logrado su progreso más notable en el campo de los negocios, la función contable es vital en todas las unidades de nuestra sociedad.

Las personas que reciben los informes contables se denominan usuarios de la información contable.

Un gerente comercial u otra persona que esté en posición de tomar decisiones y que carezca de conocimientos de contabilidad, probablemente no apreciara hasta qué punto la información contable se basa en estimativos más que en mediciones precisas y exactas.

\section{Control de Inventarios}

Con el objetivo de crear un layout eficiente en el nuevo almacén, es decir designar a cada producto un área y una ubicación conveniente se analizaran las demandas que ha tenido cada artículo por parte del departamento de producción de una empresa hipotética de acero. Creemos de vital importancia para el buen manejo de un almacén llevar el control de las salidas de los artículos, esto con el fin de transformar los datos en la información necesaria para mantener un determinado inventario de cada producto.

\section{Presentación de los datos}

La empresa lleva registro de los datos de salida de forma mensual, al capturar los datos se pone como fecha de salida el día en que se captura la información, ya que esto lo realizan mensualmente existe una relación mensual acertada de los datos de salida pero no se puede determinar el día exacto en que se generó la demanda, esto representa un problema para determinar la demanda diaria de cada producto y repercutirá en nuestro modelo.

Esta política de captura de los datos se corrigió en el mes de febrero del último año. Esto sumado al hecho de contar con un registro de los datos mayor al que se nos ha proporcionado, el cual solo consiste en un año servirá para respaldar de mejor forma los métodos de control de inventario que nosotros proponemos, así pues los resultados que genere nuestro modelo de inventarios serán solo una aproximación inicial al estudio del fenómeno que nos servirán como una referencia para determinar las cantidades de cada artículo en el almacén.

\section{Objetivos del inventario}

Los objetivos que perseguimos al mantener cierta cantidad de inventarios son los siguientes:

Garantizar el abastecimiento de materia prima a la colada continua. Este objetivo representa una prioridad pues en los procesos continuos se debe mantener la producción las 24 horas para evitar cierres y arranques costosos.

Asegurar que las materias primas no hayan excedido el tiempo recomendado para su utilización (artículos con caducidad). La empresa ha sufrido graves pérdidas por que compran materia prima en exceso, la cual en algunos casos se coloca a la intemperie en la parte posterior del almacén y como son materiales higroscópicos sufren daños irreversibles.

Asegurar la existencia de Materiales Auxiliares, los cuales sirven para la reparación de las ollas en donde se realiza la fundición. El no contar con la suficiente cantidad de estos materiales representa no poder producir ningún tipo de producto.

Disminuir los costos de ordenar y de almacenamiento.

\section{Modelos de inventarios}

Determinar la cantidad necesaria que debemos tener en inventarios no es una tarea sencilla, pues involucra muchos factores como la demanda, los costos asociados al almacén y la disposición de cada material, en nuestro objeto de estudio la cantidad que se tiene en el almacén ha sido determinada por experiencia de los encargados del departamento de compras, no ha sido planificada y es afectada por la escasez de recursos y las capacidades de almacenamiento, esto genera un círculo vicioso pues al no tener un método de control de inventarios encarecen la actividad de suministrar materia prima y productos auxiliares a acería.

\section{MARCO CONCEPTUAL}

Actividades.- Conjunto de acciones necesarias para mantener en forma permanente y continua la operatividad de las actividades de gobierno. Las actividades forman parte de un programa presupuestal.

Actividades de control.- Acciones que realiza la gerencia y otro personal de la entidad para cumplir diariamente con las funciones asignadas.

Adquisición económica.- Implica adquirir los bienes y/o servicios, en calidad y cantidad apropiada, al menor costo posible.

Ambiente de control.- Referido al establecimiento de un entorno que estimule e influencie las tareas de las personas con respecto al control de sus actividades.

Control.- Seguimiento, rastreo, alertar para asegurar que los planes y programas están siendo observados.

Control interno.- Proceso continuo realizado por la dirección y gerencia y otros empleados de la entidad, para proporcionar seguridad razonable, respecto si se están logrando los objetivos siguientes:

Promover la eficiencia, efectividad y economía en las 
operaciones y, la calidad en los servicios que debe brindar cada entidad pública.

Proteger y conservar los recursos públicos contra cualquier pérdida, despilfarro, uso indebido, irregularidad o acto ilegal.

Cumplir las leyes, reglamentos $\mathrm{y}$ otras normas gubernamentales.

Elaborar información financiera válida y confiable, presentada con oportunidad.

Estructura de control interno.- Conjunto de planes, métodos, procedimientos y otras medidas, incluyendo la actitud de la dirección de la entidad, para ofrecer seguridad razonable a se están logrando los objetivos de control interno.

Monitoreo.- Proceso que evalúa la calidad del control interno en el tiempo y permite al sistema reaccionar en forma dinámica, cambiando cuando las circunstancias así lo requieran.

Sistema integrado de información financiera.Comprende una base de datos central y única, soportada por tecnología informática y telecomunicaciones, accesible para todos los usuarios de las áreas de presupuesto, tesorería, crédito público y contabilidad gubernamental, en el nivel central y en el nivel operativo y para los administradores gubernamentales.

La aplicación del instrumento ha sido desarrollada en forma simultánea a la totalidad de los trabajadores de las empresas generadoras de tercera categoría, que representan la muestra para indagar los datos requeridos en el estudio.

El cuestionario, instrumento de la técnica de la encuesta, estuvo conformado por 10 interrogantes ordenadas y concernientes a los problemas de la investigación; cada una de las interrogantes contaba además con 2 alternativas, las cuales figuran suposiciones de aceptación o el rechazo de la interrogante planteada.

\section{Sistematización de los procesos}

\section{Interrogante:}

\section{¿Conoce usted acerca de la Sistematización de los Procesos?}

\section{Interpretación:}

Del total de personal encuestado, 60 personas, se obtuvo el siguiente resultado:

44 dijeron que "si", sumando ellos el 73,00\% del total de personas que intervinieron.

16 dijeron que "no", que alcanzaron el $27,00 \%$ del total encuestado.

De acuerdo a estos resultados, se deduce que la mayoría de personas que participaron de la presente encuesta, adujeron conocer acerca de la sistematización de los procesos y como el conjunto coordinado de los mismos facilitaría las actividades desarrolladas en el micro o pequeña empresa. La sistematización se ha ido constituyendo en una herramienta importante para el desarrollo de las actividades de una entidad, ya que sintetiza un conjunto de instrucciones que se realizan en forma constante con la finalidad de darle oportunidad inmediata a cualquier requerimiento que se exija.

Tabla 1. Sistematización de los procesos.

\begin{tabular}{|c|c|c|}
\hline Respuesta & Encuestados & $\%$ \\
\hline $\mathrm{Si}$ & 44 & $73 \%$ \\
\hline No & 16 & $27 \%$ \\
\hline Total & 60 & $100 \%$ \\
\hline
\end{tabular}

Fuente: Encuesta realizada 2014

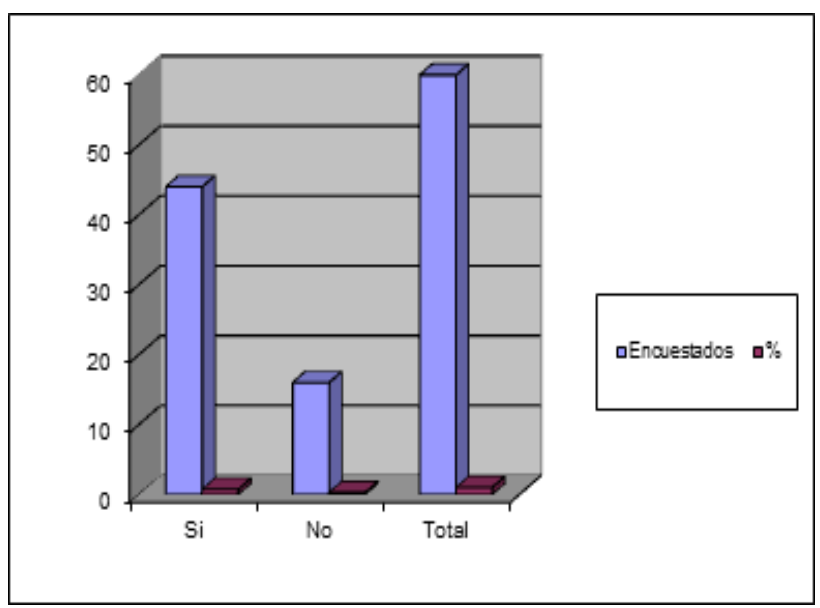

Figura 1. Sistematización de los procesos.

Fuente: Encuesta realizada 2014

Agilización de los procedimientos contables y control de inventarios

\section{Interrogante:}

¿Considera usted que mediante la Sistematización de los procesos se agilizarían los procedimientos contables y control de inventarios?

\section{Interpretación:}

De 60 personas encuestadas se obtuvo como resultados los siguientes:

39 respondieron "definitivamente sí, los cuales alcanzaron el $65.00 \%$ del total.

12 respondieron "probablemente sí", quienes fueron el $20,00 \%$ del total encuestado.

5 respondieron "probablemente no", que fueron el 8,00\% del total.

4 respondieron "definitivamente no", que sólo conformaron el $7,00 \%$ del total.

Se pudo apreciar que la mayoría de los participantes considera que la agilización de los procedimientos contables y el control de inventarios se llevaríana cabo mediante la sistematización de los mismos, toda vez 
que se estructure un sistema adecuado e integral que registre todos los procedimientos contables para que sean preparados y desarrollados con total oportunidad. Por otro lado, hubo gran cantidad de participantes que mostraron cierta duda con respecto a la sistematización de los procesos contables, esto obedece a que no tiene conocimientos acerca de cómo influiría la sistematización en la prontitud de los procedimientos.

Cuadro 2. Agilización de los procedimientos contables y control de inventarios.

\begin{tabular}{ccc}
\hline \multicolumn{1}{c}{ Categoría } & Encuestados & $\%$ \\
\hline Definitivamente si & 39 & $65 \%$ \\
Probablemente si & 12 & $20 \%$ \\
Probablemente no & 5 & $8 \%$ \\
Definitivamente no & 4 & $7 \%$ \\
\hline \multicolumn{1}{c}{ Total } & 60 & $100 \%$ \\
\hline
\end{tabular}

Fuente: Encuesta realizada 2014

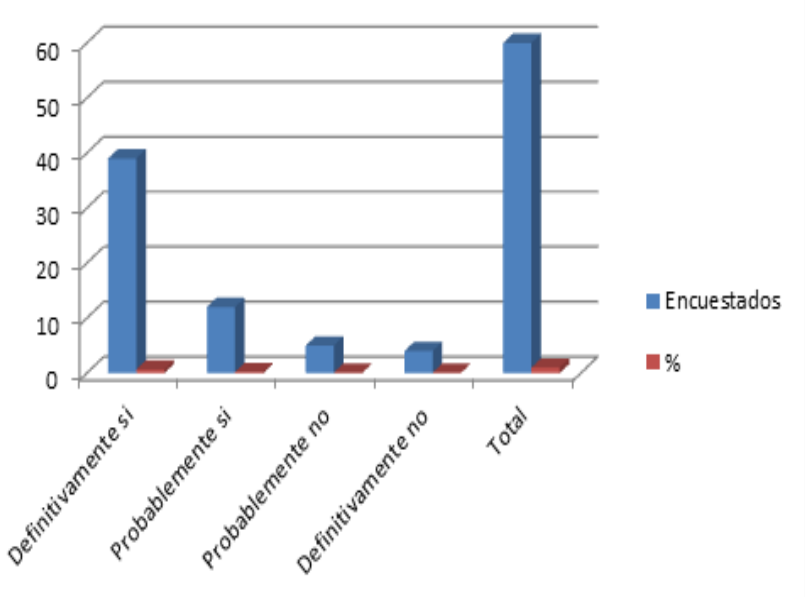

Figura 2. Agilización de los procedimientos contables y control de inventarios.

Fuente: Encuesta realizada 2014

\section{Atención de los requerimientos \\ Interrogante:}

¿Cree usted. que mediante la Sistematización de los procesos se atendería con prontitud los requerimientos de los funcionarios?

\section{Interpretación:}

De todos los encuestados, 60 personas, se muestra los resultados siguientes:

30 respondieron "completamente", y sumaron el 50,00\% del total.

26 respondieron "parcialmente", y fueron el 43,00\% del total.

04 respondieron "no", quienes fueron el resto de los encuestados, 7,00\% del total.

De acuerdo a estos resultados, se deduce que la mayoría de personas estuvieron completamente seguros que mediante la sistematización de los procesos se atenderían con oportunidad los requerimientos de los funcionarios, esto significaría un ahorro considerable del tiempo que es necesario para atender otras actividades de mayor trascendencia institucional; asimismo, se lograría descongestionar los ámbitos administrativos de control de inventarios dándole agilidad y transparencia a los engorrosos procesos existentes.

Tabla 3. Identificación de la fuente de problemas.

\begin{tabular}{lcc}
\hline \multicolumn{1}{c}{ Categoría } & Encuestados & $\%$ \\
\hline Tot. De acuerdo & 30 & $50 \%$ \\
De acuerdo & 10 & $17 \%$ \\
No sabe/No opina & 9 & $15 \%$ \\
En desacuerdo & 7 & $12 \%$ \\
Tot. En desacuerdo & 4 & $7 \%$ \\
\hline Total & 60 & $100 \%$ \\
\hline
\end{tabular}

Fuente: Encuesta realizada 2014

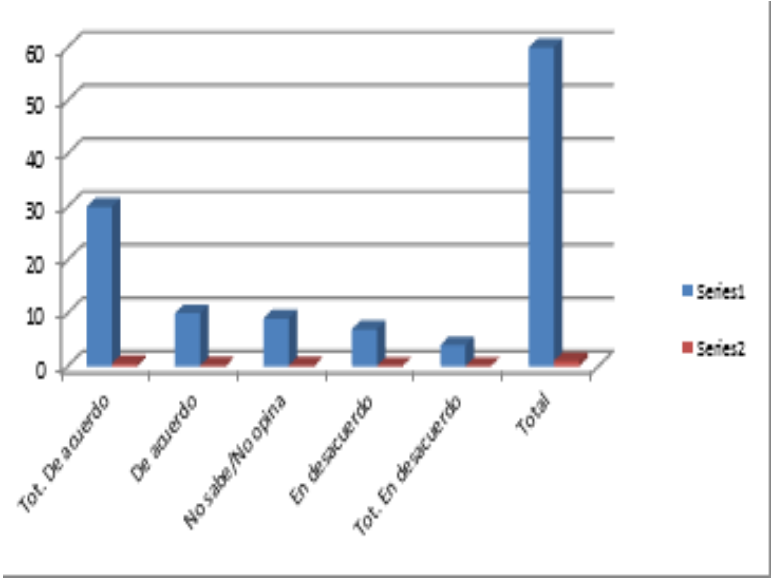

Figura 3. Identificación de las fuentes de problemas. Fuente: Encuesta realizada 2014

\section{Resolución sistematizada de los problemas Interrogante:}

¿Diga usted si mediante la Sistematización de los procesos se podrá resolver problemas prototipos o recurrentes?

\section{Interpretación:}

De todos los encuestados, 60 personas, se muestra los resultados siguientes:

33 respondieron "sí", y sumaron el 55,00\% del total.

17 respondieron "no", y fueron el $28,00 \%$ del total.

10 respondieron "no sabe / no opina", quienes fueron el resto de los encuestados, $17,00 \%$ del total.

Una gran parte de los encuestados avizoran el panorama que se tendría con la sistematización, es por eso que mediante esta interrogante, la gran mayoría se muestra 
positiva en su respuesta. Justamente la sistematización de los procesos apunta a este tipo de problemas, prototipos o ejemplares y recurrentes o reiterativos; la constancia de procesos, el mecanismo de los mismos y el poco tiempo para ejecutarlos forman la base del porqué de la sistematización; es en estos casos cuando se hace necesario la estructuración de procedimientos ya conocidos o modelos de situaciones que se han venido dando y por tal, deben estar sujeto a solución inmediata por la experiencia que se ha obtenido. Mediante la sistematización se logra analizar estos problemas logrando enmendarlos de manera eficiente y oportuna.

Tabla 4. Resolución sistematizada de los problemas.

\begin{tabular}{|c|c|c|}
\hline Respuesta & Encuestados & $\%$ \\
\hline$\overline{\mathrm{Si}}$ & 33 & $55 \%$ \\
\hline No & 17 & $28 \%$ \\
\hline No sabe/No Opina & 10 & $17 \%$ \\
\hline Total & 60 & $100 \%$ \\
\hline
\end{tabular}

Fuente: Encuesta realizada 2014

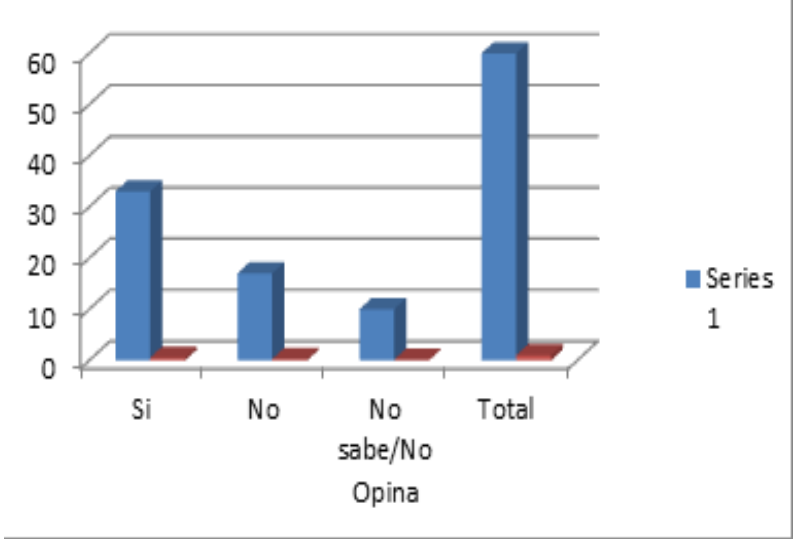

Figura 4. Resolución sistematizada de los problemas. Fuente: Encuesta realizada 2014

\section{Sistematización de cada uno de los procesos contables \\ Interrogante:}

¿Considera usted necesaria la Sistematización de cada uno de los procesos contables y control de inventarios?

\section{Interpretación:}

Del total de personal encuestado, 60 personas, se obtuvo el siguiente resultado:

36 dijeron que "sí", sumando ellos el 60,00\% del total de personas que intervinieron.

24 dijeron que "no", que alcanzaron el 40,00\% del total encuestado.

La mayoría de los encuestados considera necesaria la sistematización de los procesos a nivel de pequeñas y microempresas, debido a que, la sistematización de cada uno de los procesos de dichas organizaciones, sintetizaría un conjunto de engorrosos procedimientos de manera eficiente y eficaz, dando oportunidad al desempeño de otras labores. Por otra parte, las personas que respondieron negativamente, adujeron que la sistematización de los procesos se debe realizar de manera integral, un sólo sistema que englobe la totalidad de actividades contables a nivel de todas las áreas, toda vez que se estructure en forma adecuada uniformizando los procedimientos y la capacitación al personal de manera conjunta.

\section{Conclusiones.}

Como resultado de la investigación se ha determinado que a nivel de pequeñas y microempresas, se hace pertinente la Sistematización de los Procesos Contables con la finalidad de optimizar la administración y control de inventarios, asimismo, la eficacia, eficiencia y prontitud de los requerimientos solicitados por las distintas unidades operativas, autoridades y funcionarios, permitiendo la atención de actividades de mayor trascendencia empresarial.

Que, mediante la sistematización de los procesos contables se tiene información con oportunidad, eficacia y eficiencia, de manera que se atiendan de manera inmediata los requerimientos solicitados, reduciendo notablemente el tiempo empleado anteriormente en dicha labor.

Que, la eficiencia de la sistematización de los procesos contables logra identificar a tiempo justo las fuentes de los problemas; esta información importante constituye una ayuda significativa en la labor de los jefes, ya que podrán enmendar algunos procesos con precisión.

Que, la eficacia de la sistematización de los procesos contables logra resolver constantemente problemas prototipos o recurrentes con seguridad y prontitud, de esta manera se descongestionaría los ámbitos administrativos de control de inventarios.

Que, mediante la sistematización de los procesos contables nos permite vislumbrar con oportunidad las posibles soluciones que se pueden adoptar para un problema específico, esto ayudaría enormemente en la toma de decisiones.

Que, a través de un modelo de atención sistematizado que abarque cada uno de los procesos contables se lograría optimizar la administración y el control de inventarios a nivel de pequeñas y microempresas.

\section{Literatura citada}

Barragan, J. 2012. Administración de las Pequeñas y Medianas Empresas: retos y problemas ante la nueva economía global. México, DF. Editorial: Trillas. 147p.

Drucker, P.2007. Gestión del Conocimiento. España, Madrid. Ediciones Deusto SA. 186p.

Hidalgo, J. 2010. Auditoria de Estados Financieros. Perú, Lima. Ediciones FECAT EIRL. 233p.

Holmes, A. 1994.Auditorias Principios y Procedimientos. 
México, DF. Editorial Limusa.389p.

Meigs, J. 1994. Princípios de Auditoria.México, DF. Editorial Diana - 2da Edición. 287p.

Redondo, A.2012. Curso Práctico de Contabilidad General. Venezuela, Caracas. Editorial Centro Contable Venezolano - Décima. Edición. 163p.

Senge, P. 1998. La Quinta Disciplina.España, Barcelona. Ediciones Juan Granica SA. - Décima Edición. 490p. 